\title{
Prevalence of Testicular Artery Variations in Cadavers: Developmental Correlation and Clinical Implications
}

\author{
Saleena N. Ali, ${ }^{1}$ Tom J. Nallikuzhy, ${ }^{2}$ Suman Verma, ${ }^{3}$ Sulochana Sakthivel, ${ }^{3}$ \\ 'Department of Anatomy, P. K. Das Institute of Medical Sciences, Vaniamkulam, Kerala, India \\ ${ }^{2}$ Department of Anatomy, Sree Narayana Institute of Medical Sciences, Kunnukara, Kerala, India \\ ${ }^{3}$ Department of Anatomy, Jawaharlal Institute of Postgraduate Medical Education and Research (JIPMER), Puducherry, India
}

Disclose and conflicts of interest: none to be declared by all authors

\begin{abstract}
Introduction: Introduction The testicular arteries are liable to get injured during interventions around the renal pedicle. The present study aimed to record the anatomical variations in testicular arteries to assist surgeons and radiologists in avoiding unforeseen complications.

Materials and Methods: a total of 25 formalin-embalmed adult cadavers were dissected, and the number, origin, relationships with the inferior vena cava and renal veins of the testicular arteries were recorded. The distance between the origins of the renal and testicular arteries from the abdominal aorta was measured, and the results were statistically analyzed. Results: out of 50 cadaveric sides, variations were observed in $14 \%$. The most commonly observed variation was an arched testicular artery (10\%). Double testicular arteries were observed in $4 \%$ of the sample, and testicular arteries with renal origin were observed in $6 \%$. Most variations were right-sided (71.4\%). The testicular artery originated at the second lumbar vertebral level in $94.2 \%$ of the sample. The mean distance between the origins of the testicular and renal arteries was 3.60 $0.36 \mathrm{~cm}$ on the right, and $2.28 \pm 0.92 \mathrm{~cm}$ on the left, and the difference between the two sides was statistically significant. Conclusion: the left testicular artery is closer to the renal pedicle, and is more likely to be at risk during surgeries in the hilar area. Cases with renal origin and arching of the testicular artery are also at a high risk. Compression of the testicular artery due to arching or retrocaval course could be a causative factor for the development of varicocele. The presence of such variations should be meticulously assessed using radiological imaging prior to interventions.
\end{abstract}

Keywords: Testes blood supply; Testicular artery anomalies; Testes; Anatomic variation.

\section{Introduction}

Testicular arteries (TAs) arise as the lateral branches of the abdominal aorta (AA), inferior to the corresponding renal arteries (RAs), at the level of the second lumbar vertebra (L2). The arteries run retroperitoneally along the psoas major muscle toward the pelvis. At the deep inguinal ring (DIR), the TA becomes a content of the spermatic cord, and proceeds to the scrotum. In its course, the right TA is related posteriorly to the inferior vena cava (IVC). Though the TA supplies mainly the testes, it provides numerous small branches to the ureter and epididymis as well. The artery enters the testes through the mediastinum and branches in the tunica vasculosa. ${ }^{1}$

However, variations in the origin and course of the TA are not uncommon, and Pai et $a l^{2}$ (2008) repored a $14.7 \%$ incidence of such variations. Inadvertent injury to the TA due to unsuspected variation can lead to intraoperative hemorrhage and can even be fatal. Apart from the complications related to the incidental findings, the variations in the TA might alter the thermoregulation and thus affect the normal physiological functions of the testes. ${ }^{3}$ Animal studies have proven that a reduction in testicular blood flow could result in spermatogenic arrest and alter the levels of testosterone in the blood. ${ }^{4}$

The close relationship of the TA with the renal pedicle poses a high risk of injury to the vessels during surgeries in the renal hilar area. ${ }^{3}$ As the demand for renal transplants is currently increasing, a precise and thorough knowledge regarding the anatomical variations in the purlieu of the renal hilar area is essential for interventional nephrologists and radiologists. It has also been postulated that such variations might play a role in the pathogenesis of varicocele that might lead to sterility. ${ }^{5,6}$ Awareness of variations becomes noteworthy during various surgical procedures such as varicocelectomy and microvascular autotransplantation of intra-abdominal testis. ${ }^{7}$

Shoja et $a l^{8}$ (2007) reported the incidence of TAs with high origin (superior to L2) as between 5\% and 6\%. Cauldwell and Anson9 (1943) observed that 74\% of right TAs originated at the vertebral level of L2-L3, and $80 \%$ of left TAs originated at the lower third of L2 vertebra, evidencing a higher level of origin of left TAs from the AA just below that of the RA. However, there 
is a lack of sufficient literature regarding the exact distance between the two vessels. Hence, the present observational study was proposed to determine the anatom-ical variations in the TA, as well as to measure the distance between the origin of the TA and RA from the AA, which might prove useful for surgeons and interventional radiol-ogists to avoid various complications.

\section{Materials and Methods}

By using the convenient sampling technique, 25 formalin-embalmed adult male cadavers (age: 5570 years), available at the Department of Anatomy during the period of 3 years (2016 to 2018) were selected. These specimens were retrieved from the institutional body donation program following the ethical guidelines, and were preserved in $10 \%$ formalin after embalming. The cadavers with signs of visible damage in and around the area of dissection were excluded. The dissection of the abdominal cavities was performed as per the Cunningham manual of dissection, ${ }^{10}$ and the retroperitoneal structures were exposed. The connective tissue surrounding the AA, the testicular vessels, the IVC, the renal vessels and the kidneys was cleared, and the TA was traced from its origin to the DIR.

The number of TAs, their origin, course, distance from the midpoint of the ipsilateral RA, and relation with the IVC and the renal vein were observed. All of the dissected specimens were photographed with a digital camera. The distance from the RA was measured with a digital Vernier calliper (Mitutoyo, Tokyo, Japan) to the nearest millimeter. The measurements were obtained by a single investigator thrice, and the mean of the three readings was taken as the final measurement. The statistical analysis was performed using the GraphPad QuickCalcs software (GraphPad Software, San Diego, CA, US), and $p$-values $<0.05$ were considered statistically significant.

\section{Results}

In the 50 sides examined, the TA was variable in $14 \%$. The four types of variations observed were: double TA, aberrant origin of TA, TA arching over the renal vein, and retrocaval TA. Arched TA (10\%) was the most common type of variation encountered, followed by aberrant origin (8\%). Double TAs were present in $4 \%$, and the retrocaval course was observed in $6 \%$ of the sample. The RA and suprarenal artery (SRA) were the two sources for the aberrant origin (Fig. 1, 2), the former being the most frequent (6\%).

The distribution of variations on the right and left sides is shown in Table 1. The right-sided variations were more common (71.4\%). The aberrant origin was right-sided in $75 \%$, the double and retrocaval TAs were always right-sided in presentation, where as the arched TA (Fig. 3) was left-sided in $60 \%$ of the sample. In the two cases with double arteries, the superior arteries were both retrocaval and arched. The superior artery (Fig. 2) originated from the SRA or the RA, and the inferior one, from the AA, and both the arteries were traced till the testes, where they ended separately. The TA variations were bilateral in three cadavers and unilateral in one. The TA arching over the ipsilateral renal vein was bilateral in two and unilateral in one. All of the other types of variations were unilateral in appearance.

The TA was below the RA in $92.3 \%$, and above it in $1.9 \%$ of the sample. In cases of origin of the TA from the AA, the distance between the origins of the TA and the ipsilateral RA from the AA was measured and summarized, as seen in Table 2. The difference in the means of both sides was found to be statistically significant. The highest level of origin was observed in $3.84 \%$ of the sample at the disc between the L1 and L2 vertebrae. The lowest level of origin at the L3 vertebra was present in $1.9 \%$ of the sample.

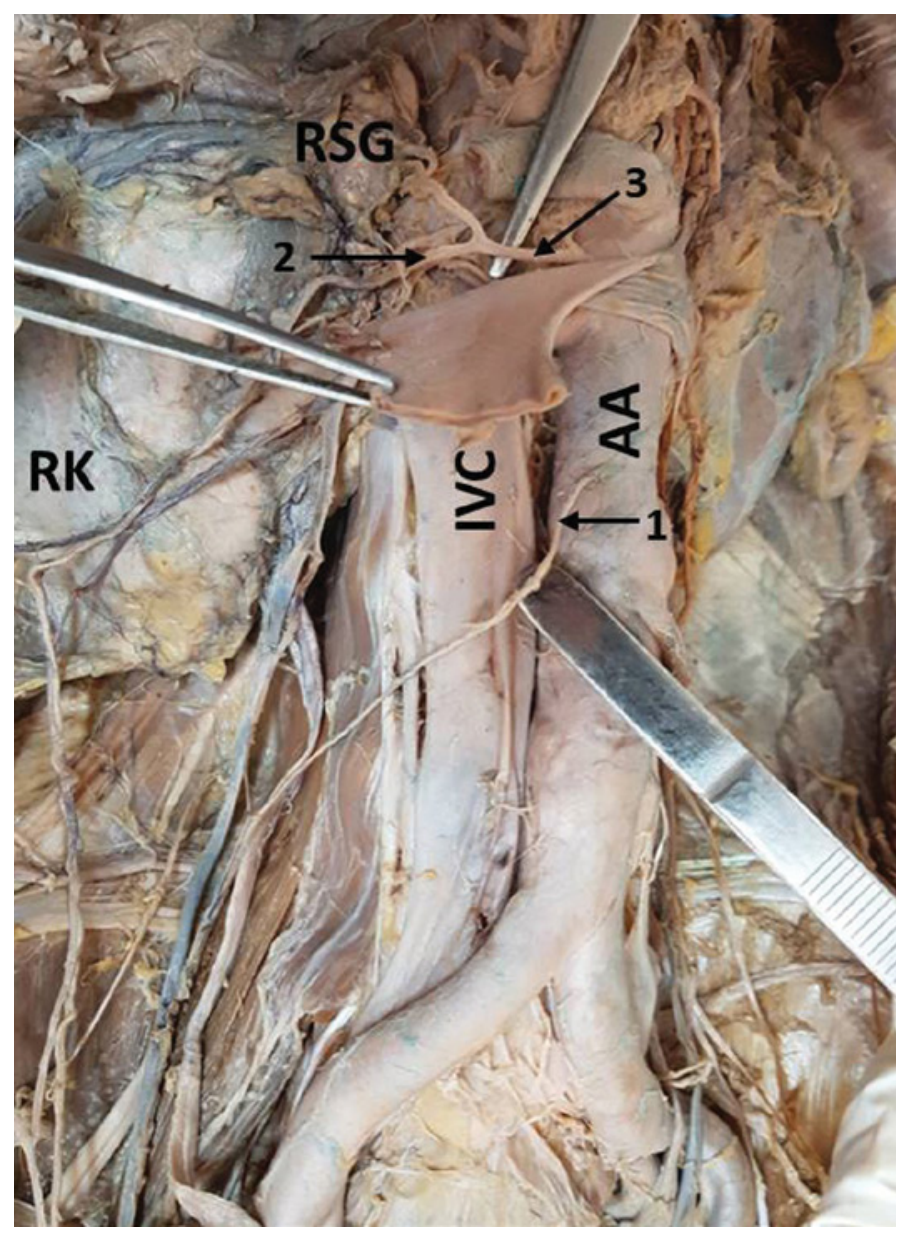

Figure 1. Double testicular arteries (right). 1, inferior testicular artery; 2, superior testicular artery; 3, middle suprarenal artery; AA, abdominal aorta; IVC, inferior vena cava; RK, right kidney; RSG, right suprarenal gland. 


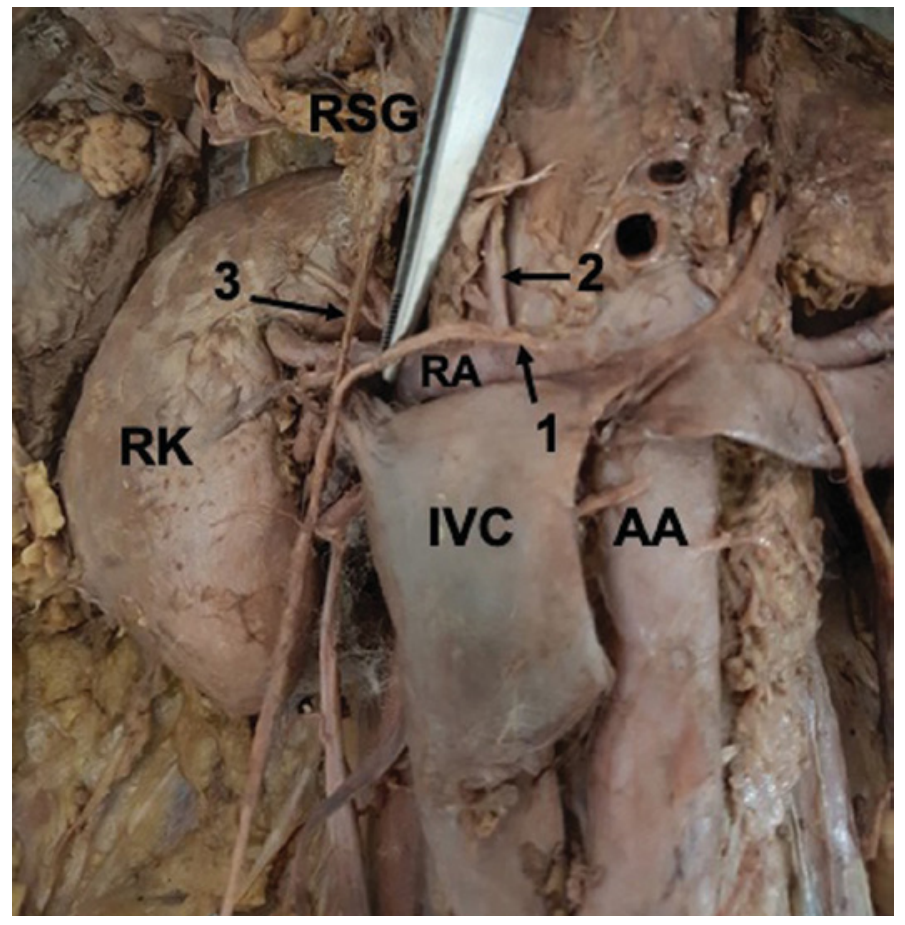

Figure 2. Aberrant renal origin of the testicular artery (right). 1, testicular artery; 2, right inferior suprarenal artery; 3, a branch from the testicular artery to suprarenal gland; AA, abdominal aorta; IVC, inferior vena cava; RK. right kidney; RSG, right suprarenal gland.

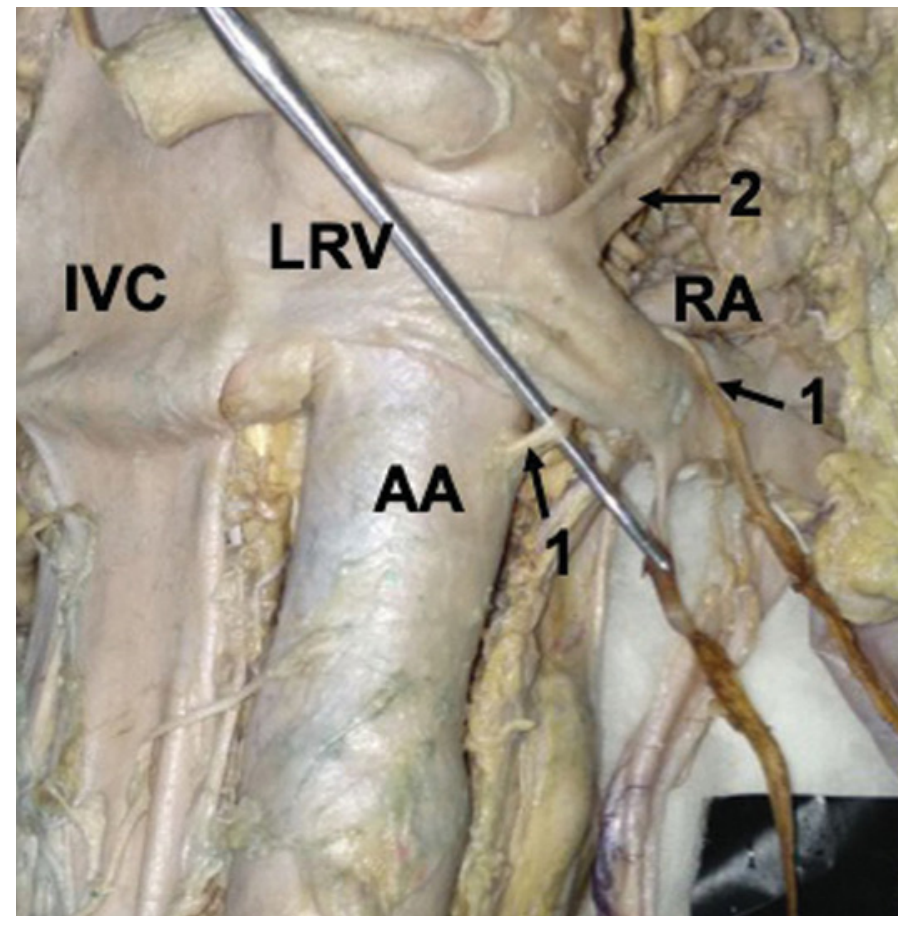

Figure 3. Arched testicular artery (left). 1, testicular artery; 2, left suprarenal vein; AA, abdominal aorta; IVC, inferior vena cava; RA, renal artery; LRV, left renal vein.

Table 1. Distribution of various types of TA variations

\begin{tabular}{l|c|c|c|c|c|c|c|c|c}
\hline & \multicolumn{2}{|c|}{ Number (\%) } & \multicolumn{3}{c|}{ Origin (\%) } & \multicolumn{4}{c}{ Course (\%) } \\
\cline { 2 - 10 } & Single & Double & AA & RA & SRA & $\begin{array}{c}\text { Retrocaval } \\
\text { only }\end{array}$ & Arched only & $\begin{array}{c}\text { Retrocaval and } \\
\text { arched }\end{array}$ & Normal \\
\hline Right & $23(92)$ & $2(8)$ & $23(85.2)$ & $3(11.1)$ & $1(3.7)$ & $1(3.7)$ & 0 & $2(7.4)$ & $24(88.9)$ \\
\hline Left & $25(100)$ & 0 & $24(96)$ & $1(4)$ & 0 & 0 & $3(12)$ & 0 & $22(88)$ \\
\hline
\end{tabular}

Abbreviations: AA, abdominal aorta; RA, renal artery; SRA, suprarenal artery; TA, testicu

Table 2. Distance between the origins of the renal artery and testicular artery

\begin{tabular}{l|c|c|c|c|c}
\hline $\begin{array}{c}\text { Distance from the } \\
\text { renal artery }\end{array}$ & Minimum & Maximum & Mean (cm) & Standard deviation $^{\boldsymbol{p}_{\text {-value }}^{\#}}$ \\
\hline Right & 2.77 & 4.07 & 3.60 & 0.36 & $<0.001$ \\
\hline Left & 0.8 & 4.01 & 2.28 & 0.92 & \\
\hline
\end{tabular}

Note: "Paired t test.

\section{Discussion}

Variations related to the origin of the TA, such as number, source and level of origin from the AA, have been reported to occur at a rate of $4.7 \% .^{11}$ With respect to the number of TAs, double TA is more common compared with the multiple TA or absent TA. ${ }^{12}$ The incidence of unilateral double TA varies widely between $1.4 \%$ and $20 \%,{ }^{13}$ which is comparable to the results of the present study (4\%). Pai et al ${ }^{2}$ (2008) reported an incidence of double TA in $1.5 \%$ of the Southern Indian population, and Wadhwa and Soni ${ }^{14}$ (2010) reported a similar incidence (1.7\%) in Northern India. In accordance with the present study, both of these studies reported the double TA on the right side. In "Bergman's Comprehensive Encyclopedia of Human
Anatomic Variation," Tubbs et $a^{15}$ (2016) reported that among the right-sided double TAs, the accessory TA originated from the AA (10.52\%), the RA (2.63\%), and the accessory RAs (2.63\%). In the double TAs in the present study, the accessory TA originated from the RA and the middle SRA. The presence of double TA should be diagnosed before any interventions on the testes. Incomplete ligation and failure of the treatment in cases of orchiectomy or testicular carcinoma can occur due to unfamiliarity with such variations. ${ }^{14}$ The possibility of origin of the TA from the SRA should be kept in mind during the laparoscopic adre-nalectomy and treatment of adrenal artery aneurysms. ${ }^{16}$

Asala et $a l^{11}$ (2001) and Wadhwa and Soni1 ${ }^{14}$ (2010) observed the renal origin in $3.4 \%$ and $5 \%$ of their 
samples respectively. A radiographic study ${ }^{3}$ revealed that $14 \%$ of donated kidneys had the gonadal artery originating from the RA. We observed the renal origin of the TA in $6 \%$ of the cases; however, the incidence of renal origin of the TA ranges from $1.5 \%$ to $16.3 \%{ }^{2,13}$ The renal origin makes the TA a part of the renal pedicle, complicating various surgeries, such as nephrectomies, renal transplantation, angioplasties and percutaneous surgery for ureteropelvic junction obstruction. ${ }^{17}$ Testicular infarction may also result from the transcatheter embolization of a malignant renal tumor. ${ }^{18}$

Though it is well known that the TA arises from the AA below the ipsilateral RA, few studies have reported the exact distance between the origins of the two arteries. Kotian et $a^{19}$ (2016) reported the mean distance between the origin of the TA and the RA as $3.08 \mathrm{~cm}$ on the right side and $3.49 \mathrm{~cm}$ on the left. In the present study, the distance between the origin of the left TA and the RA was significantly shorter as compared with that on the right side. Based on our findings, we propose that the left TA is more likely to get injured while exploring the renal hilar area or performing angioplasties due to its closeness to the renal pedicle. With respect to the relation of the TA to the renal veins, Notkovich ${ }^{20}(1955)$ classified the TA into three types, and the arched TA was the third variety. The author observed $8(16 \%)$ arched TAs on the left side, whereas only 2 (4\%) on the right side..$^{20}$ Pai et al ${ }^{2}(2008)$ and Naito et $a^{21}$ (2006) observed a similar variation in $4.4 \%$ and $6.7 \%$ of their samples respectively, and in both studies the authors noted cases on the left side. Adebisi and Singh22 (2000) reported a high prevalence of arched left TAs (64\%) compared with those on the right side (52\%) in the Nigerian population, which is similar to our results..$^{22}$ The higher level of origin of left TAs from the AA compared with the right side, as observed in the present study, may explain the higher prevalence of left arched TAs.

The TA becomes a part of the renal pedicle when it originates from the RA, or in the presence of an arched TA. Precise knowledge of such variations is crucial for the surgeons while exploring the renal hilar area, as well as for the interventional radiologists performing angioplasties. Moreover, such possible variations should be kept in mind during the treatments, like that for the undescended testes and torsion of testes. ${ }^{3,23}$ The downward traction, as well as the compression of the renal vein due to the presence of an arched TA can result in poor venous drainage from the kidney and left testis. Hence, such compression may be a causative factor for the development of varicocele, orthostatic albuminuria, and even testicular atrophy. ${ }^{5,20}$ Tarhan et al (2003) reported that the testicular blood flow was significantly reduced in patients with varicocele, resulting in impaired spermatogenesis. ${ }^{24}$ Compression of the left renal vein between the $\mathrm{AA}$ and the superior mesenteric artery results in the nutcracker phenomenon, which leads to left renal hypertension and hematuria. ${ }^{25}$ This condition can be mimicked in arched TAs as well. ${ }^{5}$ The retrocaval course was observed in $4 \%$ of the sample by Notkovich ${ }^{20}$ (1955), whereas, we found it in $6 \%$ of our sample. However, the prevalence of the retrocaval course has been reported as low as $0.67 \%,{ }^{11}$ and as high as $10 \% .{ }^{14}$ Such a variation can lead to the compression of the TA under the IVC during increased venous return, which can impede the venous drainage and, thus, lead to stasis and even varicocele. ${ }^{23}$

The variations in origin and course of the TA can be attributed to the embryonic development. Felix, ${ }^{26}$ in 1912, was the first to describe the development of the TA. The lateral mesonephric arteries (LMAs) arise as lateral branches from the dorsal aorta, and are arranged as the cranial, middle and caudal groups. These arteries supply the kidneys, suprarenal glands, testes, diaphragm, lymph nodes, and sympathetic ganglia. Initially, around 30 LMAs exist, but, later, most of them regress, and only 9 to 11 arteries persist in the lower thoracic and upper lumbar regions. The cranial group comprises the first and second LMAs at the level of the celiac trunk, the middle group is composed of the third to fifth LMAs, and the caudal group includes the sixth to ninth LMAs. Since the testes are positioned at a higher level during the early period of development, they are initially supplied by the LMAs of the cranial group. Later, as the testes descend, one pair of the caudal group of arteries persists as the TA and supplies the organ. Apart from the TA, the LMAs also give rise to phrenic arteries SRA, RA and ARA. Felix ${ }^{26}$ also proposed that when the TA had a higher origin from the cranial group of LMAs, it would be posterior to the RA that probably arises from the middle group. ${ }^{26}$

Ciçekcibaşi et $a^{27}$ (2002) revealed that the cranial group of LMAs gave rise to a gonadal artery that originated from the SRA or a higher level, while the middle group of LMAs gave rise to gonadal arteries from the RA. Since all LMAs are connected to each other by the longitudinal anastomotic channels, the possibility of the TA, RA or SRA having a common trunk from the AA, and the TA with the higher origin being anterior to the RA cannot be ruled out. ${ }^{3}$ A high origin of the TA might be due to the persistence of a cranial LMA that supplied the gonad before it descended to the scrotal sac. On the other hand, failure of regression of any of the middle or cranial groups of LMAs along with persistence of the caudal group of arteries might be the cause for the presence of double TA in the present study. Persistence of the caudal group of LMAs below the renal pedicle along with a high ascent of the kidney could result in an arched TA. ${ }^{26}$

The retrocaval course would be the result of variations in the development of the IVC rather than the TA. Under normal circumstances, the IVC develops from multiple sources. The renal segment develops from the anastomosis between the right supracardinal 
and subcardinal veins. The retrocaval course of the TA results when it courses cranially to the anastomosis, instead of being caudal to it. ${ }^{28}$

\section{Conclusion}

As per the present study, in the Southern Indian population, the RA is the most common source of aberrant origin of the TA. Moreover, the left TA is closer to the renal pedicle, and, hence it is more likely to get injured. Based on our observations, an arched TA on the left side is the most common variant that can be encountered, and should always be ruled out in cases of varicocele. The presence of such variations should be meticulously assessed using any radiological imaging techniques, such as angiography, Doppler ultrasound or contrastenhanced computed tomography, so as to prevent unforeseen complications.

\section{References}

1. Goldstein M, Mehta A. Male Reproductive System. In: Standring S, Gray H. eds. Gray's anatomy: the anatomical basis of clinical practice, 41 $1^{\text {st }}$ ed. Philadelphia: Elsevier; 2016:1272-1287.

2. Pai MM, Vadgaonkar $\mathrm{R}$, Rai $\mathrm{R}$, et al. A cadaveric study of the testicular artery in the South Indian population. Singapore Med J 2008;49(07):551-555.

3.Paraskevas GK, Ioannidis O, Raikos A, et al. High origin of a testicular artery: a case report and review of the literature. J Med Case Reports 2011;5:75.

4. Hsu TH, Huang JK, Ho DM, Liu RS, Chen MT, Chang LS. Role of the spermatic artery in spermatogenesis and sex hormone synthesis. Arch Androl 1993;31(03):191-197

5. Ranade AV, Rai R, Prahbu LV, Mangala K, Nayak SR. Arched left gonadal artery over the left renal vein associated with double left renal artery. Singapore Med J 2007;48(12):e332-e334.

6. Eisenberg ML, Lipshultz LI. Varicocele-induced infertility: Newer insights into its pathophysiology. Indian J Urol 2011;27(01): 58-64.

7. Bukowski TP, Wacksman J, Billmire DA, Sheldon CA. Testicular autotransplantation for the intra-abdominal testis. Microsurgery 1995;16(05):290-295.

8. Shoja MM, Tubbs RS, Shakeri AB, Oakes WJ. Origins of the gonadal artery: embryologic implications. Clin Anat 2007;20(04):428-432.

9. Cauldwell EW, Anson BJ. The visceral branches of the abdominal aorta: topographical relationships. Am J Anat 1943;73:27-57.

10. Romanes GJ. Cunningham's manual of practical anatomy. New York: Oxford University Press; 1986:113-180.

11. Asala S, Chaudhary SC, Masumbuko-Kahamba N, Bidmos M. Anatomical variations in the human testicular blood vessels. Ann Anat 2001;183(06):545-549.

12. Nallikuzhy TJ, Rajasekhar SSSN, Malik S, Tamgire DW, Johnson $P$, Aravindhan K. Variations of the testicular artery and vein: A metaanalysis with proposed classification. Clin Anat 2018;31(06):854-869

13. Paraskevas GK, Natsis K, Nitsa Z, Papaziogas B, Kitsoulis P. Bilateral double testicular arteries: a case report and review of the literature. Potential embryological and surgical considerations. Folia Morphol (Warsz) 2014;73(03):383-388.

14. Wadhwa A, Soni S. A study of gonadal arteries in 30 adult human cadavers. Clin Med Insights Reprod Health 2010;4:1-5.

15. Tubbs RS, Shoja MM, Loukas M, Eds. Bergman's Comprehensive Encyclopedia of Human Anatomic Variation. Hoboken, New Jersey: John Wiley \& Sons, Inc; 2016:1432.
16. Jyothsna P, Mohandas Rao K, Somayaji S, Ashwini L. Multiple vascular anomalies involving testicular, suprarenal arteries and lumbar veins. N Am J Med Sci 2012;4(03):154-156.

17. Ravery V, Cussenot O, Desgrandchamps F, et al. Variations in arterial blood supply and the risk of hemorrhage during percutaneous treatment of lesions of the pelviureteral junction obstruction: report of a case of testicular artery arising from an inferior polar renal artery. Surg Radiol Anat 1993;15(04):355-359.

18. Siniluoto TM, Hellström PA, Päivänsalo MJ, Leinonen AS. Testicular infarction following ethanol embolization of a renal neoplasm. Cardiovasc Intervent Radiol 1988;11(03):162-164.

19. Kotian SR, Pandey AK, Padmashali S, Jaison J, Kalthur SG. A cadaveric study of the testicular artery and its clinical significance. J Vasc Bras 2016;15(04):280-286.

20. Notkovich $\mathrm{H}$. Testicular artery arching over renal vein: clinical and pathological considerations with special reference to varicocele. $\mathrm{Br}$ J Urol 1955;27(03):267-271.

21. Naito M, Terayama H, Nakamura Y, Hayashi S, Miyaki T, Itoh M. Left testicular artery arching over the ipsilateral renal vein. Asian J Androl 2006;8(01):107-110.

22. Adebisi SS, Singh SP. Anomalous gonadal arteries in relation to the renal vein: a preliminary study in Nigerians. Niger J Surg Res 2000;2(3-4):148-151.

23. Mamatha H, D'Souza AS, Vinodhini P, Ray B. Suhani, Pallavi. A cadaveric study about the anomalous origin of testicular arteries arising from the accessory renal arteries. Indian J Surg 2015;77 (02):111-116

24. Tarhan S, Gümüs B, Gündüz I, Ayyildiz V, Göktan C. Effect of varicocele on testicular artery blood flow in men-color Doppler investigation. Scand J Urol Nephrol 2003;37(01):38-42

25. Nickavar A. Nutcracker syndrome; a rare cause of hematuria. J Nephropathol 2016;5(04):144-145.

26. Felix W. Mesonephric arteries (aa. mesonephricae). In: Keibel F, Mall FP (eds) Manual of Human Embryology Volume 2. Phila- delphia: Lippincott; 1912:820-825.

27. Ciçekcibaşi AE, Salbacak A, Şeker M, Ziylan T, Büyükmumcu M,

Uysal ii. The origin of gonadal arteries in human fetuses: anatomical variations. Ann Anat 2002;184(03):275-279.

28. Lelli F, Maurelli V, Maranillo E, Valderrama-Canales FJ. Arched and retrocaval testicular arteries: a case report. Eur J Anat 2007;11 (02):119-122.
Received: July 10, 2019

accepted: September 4, 2019
Corresponding author

Suman Verma

Email: suman2v@gmail.com 